\title{
DISCOVERY OF VARIABLE IRON FLUORESCENCE FROM REFLECTION NEBULAE IN THE GALACTIC CENTER
}

\author{
M. P. Muno, ${ }^{1}$ F. K. Baganoff, ${ }^{2}$ W. N. Brandt, ${ }^{3}$ S. Park, ${ }^{3}$ and M. R. Morris ${ }^{4}$ \\ Received 2006 November 2; accepted 2007 January 4; published 2007 January 31
}

\begin{abstract}
On the basis of 3 years of deep observations of the Galactic center with the Chandra X-Ray Observatory, we report the discovery of changes in the intensities and morphologies of two hard X-ray nebulosities. The nebulosities are dominated by fluorescent iron emission and are coincident with molecular clouds. The morphological changes are manifest on parsec scales, which requires that these iron features are scattered X-rays from a 2 or 3 year long outburst of a point source (either Sgr $\mathrm{A}^{*}$ or an X-ray binary) with a luminosity of at least $10^{37} \mathrm{ergs} \mathrm{s}^{-1}$. The variability precludes the hypotheses that these nebulae either are produced by $\mathrm{keV}$ electrons bombarding molecular clouds or are iron-rich ejecta from supernovae. Moreover, the morphologies of the reflection nebulae implies that the dense regions of the clouds are filamentary, with widths of $\approx 0.3 \mathrm{pc}$ and lengths of $\approx 2 \mathrm{pc}$.
\end{abstract}

Subject headings: Galaxy: center — ISM: clouds - X-rays: ISM

\section{INTRODUCTION}

As a result of the high density of matter and stars in the Galactic center, opportunities arise for energetic particles, radiation, and interstellar matter to interact in ways that occur very rarely in the Galactic disk. One manifestation of these interactions is iron fluorescence nebulae that are visible in the $\mathrm{X}$-ray band and that are associated with the molecular cloud complexes Sgr B1 and B2 (Murakami et al. 2000, 2001b), Sgr C (Murakami et al. 2001a), and M0.11-0.11 (Park et al. 2004). The fluorescence is thought to be produced when lowionization iron in the molecular clouds is bombarded either by $\sim 30 \mathrm{keV}$ electrons (Valinia et al. 2000; Yusef-Zadeh et al. 2002; Bykov 2002) or by $\sim 8 \mathrm{keV}$ X-rays (Sunyaev \& Churazov 1998; Revnivtsev et al. 2004b). However, exciting the fluorescent features with electrons would require an arguably unreasonable amount of energy in keV electrons (Revnivtsev et al. 2004b), and there is currently no X-ray source in the Galactic center bright enough to illuminate these features (Sunyaev et al. 1993; Koyama et al. 1996; Revnivtsev et al. 2004a). Therefore, the excitation mechanism for these features is uncertain.

In this Letter we address how the iron fluorescence features are excited by searching for variations in the morphologies and intensities of the two examples with the highest surface brightnesses (features 1 and 2 in Park et al. 2004), which are located in the M0.11-0.11 cloud complex. If the fluorescence is excited by particles, which would propagate rather slowly $(<0.06 c$ for $30 \mathrm{keV}$ electrons), the features would not vary over the course of a few years. Alternatively, if the fluorescence is produced by X-rays, they would have to have been produced in a transient event. If the outburst were short enough, then in a few years the light front could propagate across the features, causing their morphologies and intensities to change (Sunyaev \& Churazov 1998). Here we report that, in fact, the two fluorescent features have changed after 3 years.

\footnotetext{
${ }^{1}$ Space Radiation Laboratory, California Institute of Technology, Pasadena, CA; mmuno@srl.caltech.edu.

${ }^{2}$ Kavli Institute for Astrophysics and Space Research, Massachusetts Institute of Technology, Cambridge, MA; fkb@space.mit.edu.

${ }^{3}$ Department of Astronomy and Astrophysics, Pennsylvania State University, University Park, PA; niel@astro.psu.edu, park@astro.psu.edu.

${ }^{4}$ Department of Physics and Astronomy, University of California, Los Angeles, CA; morris@astro.ucla.edu.
}

\section{OBSERVATIONS}

The features that we studied were identified by Park et al. (2004) based on $500 \mathrm{ks}$ of observations of the $20 \mathrm{pc}$ around Sgr A* taken with the Advanced CCD Imaging Spectrometer imaging array between 2002 May 22 and June 3. Since then, $100 \mathrm{ks}$ of data were acquired between 2004 July 5 and 6, and 200 ks between 2005 July 24 and August 1 . All of the observations had aim points within $20^{\prime \prime}$ of $\mathrm{Sgr} \mathrm{A}^{*}$, although the roll angles for the satellite differed in $2002\left(76^{\circ}\right)$ and 2004-2005 $\left(280^{\circ}\right)$. Features 1 and 2 are located near $\alpha=266.492^{\circ}$, $\delta=-28.943^{\circ}(\mathrm{J} 2000.0)$, or $6^{\prime}$ from Sgr $\mathrm{A}^{*}$, and are covered by all of our observations. At this offset, the point-spread function (PSF) at $6 \mathrm{keV}$ has a FWHM of 6", which corresponds to a physical scale of $0.2 \mathrm{pc}(D=8 \mathrm{kpc}$; McNamara et al. 2000).

We processed the event lists from each observation to remove bad pixels, cosmic rays, and background flares in the standard manner, ${ }^{5}$ using CIAO version 3.3.0 and the calibration database version 3.2.2. We identified pointlike X-ray sources using the wavelet algorithm wavdetect. The absolute astrometric frame was registered using X-ray sources with counterparts in the 2MASS catalog (Skrutskie et al. 2006), making it accurate to $0.2^{\prime \prime}$.

To search for temporal evolution, we produced three separate images of the flux in the $4-8 \mathrm{keV}$ band, using the data from 2002, 2004, and 2005. This limited energy range was chosen to reject foreground emission, emission from soft plasma, and particle background. In Figure 1 we display the portion of the field containing features 1 and 2, smoothed by a Gaussian with a FWHM of 6 . Having smoothed the images on this scale, changes in the PSF shape are not significant, as can be seen by examining point sources in the image. Over 3 years, the morphologies of features 1 and 2 change noticeably.

These variations can be seen more clearly in difference images normalized to the uncertainty in the convolved flux (Fig. 2). To generate these, we first calculated the one-sided, upper $1 \sigma$ Poisson uncertainty on the counts in each pixel of each image, following Gehrels (1986). We divided this by the corresponding exposure maps to obtain the uncertainties on the flux, $\sigma_{f, i}$. To get the uncertainties in each convolved image $\left(\sigma_{C, i}\right)$, we convolved the square of the uncertainties with the square of the Gaussian smoothing function $(g), \sigma_{C, i}=$ $\left(\sigma_{f}^{2} * g^{2}\right)_{i}^{1 / 2}$. The uncertainties in each pair of images were then

\footnotetext{
${ }^{5}$ See http://cxc.harvard.edu/ciao/threads/.
} 


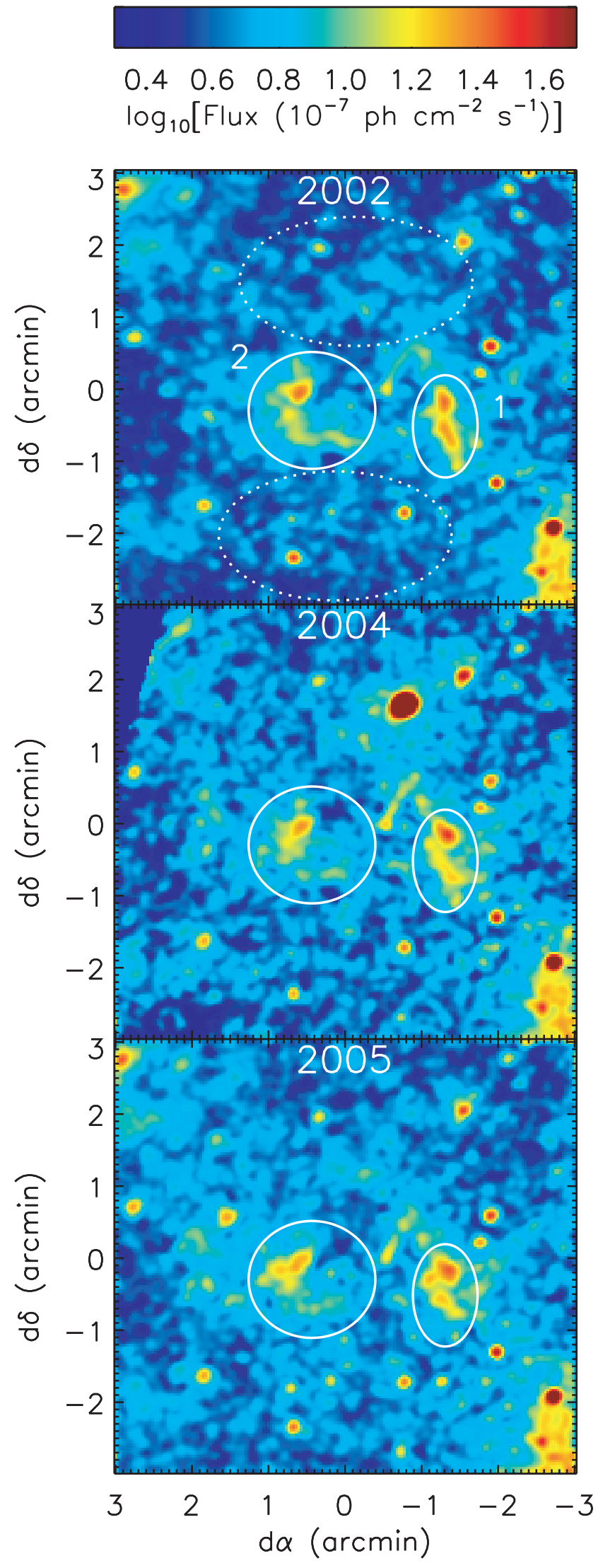

FIG. 1.-Hard (4-8 keV) X-ray images of the $6^{\prime} \times 6^{\prime}$ field around the iron fluorescent features (centered at $\alpha, \delta=266.44156,-28.94267$ [J2000.0]), illustrating their evolution as a function of time. The solid ellipses denote the locations of the fluorescent iron features from Park et al. (2004). The dashed ellipses in the top panel denote the regions used for background extraction. The bar at top displays the mapping between flux and color, with red indicating the brightest emission, and blue the faintest.

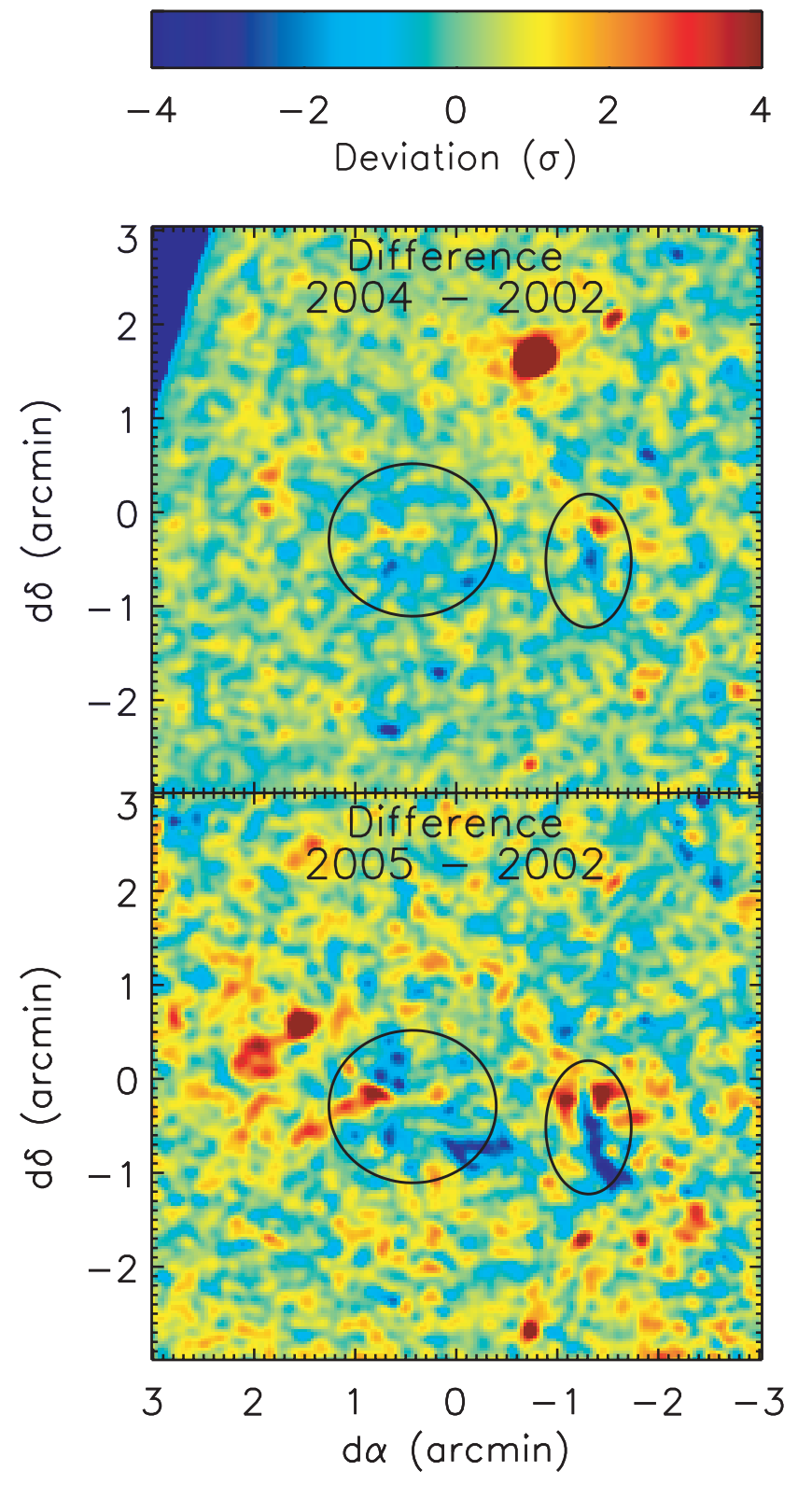

FIG. 2.-Significance maps of the difference in flux received in the 4$8 \mathrm{keV}$ band. Red indicates an increase in intensity. At the locations of the fluorescent features, the variations in flux are spatially coherent, indicating that the diffuse features have changed in shape and intensity. The changes are more significant in the 2005-2002 difference map, because the exposure was twice that in 2004. The bar at the top of the figure provides the mapping between significance and color.

added in quadrature to obtain those of the difference images. The difference images were divided by the uncertainty maps to obtain the significance maps.

In Figure 2, red features were defined to be brighter in the later images; a clear example is the transient source XMM J174554.4-285456 (Porquet et al. 2005; Muno et al. 2005), which appeared in 2004 just north of the center of the image. We find significant variations in several places in the field. The main ridge that composed feature 1 in 2002 faded at the $3 \sigma$ level by 2005 , while the northern part of the feature on either side of the ridge brightened by $4 \sigma$. For feature 2 , we find that the southern portion faded by $3 \sigma$ between 2002 and 2005, whereas the eastern portion shifted farther east and became more extended at the 4-5 $\sigma$ level. We also find 3-4 $\sigma$ increases 


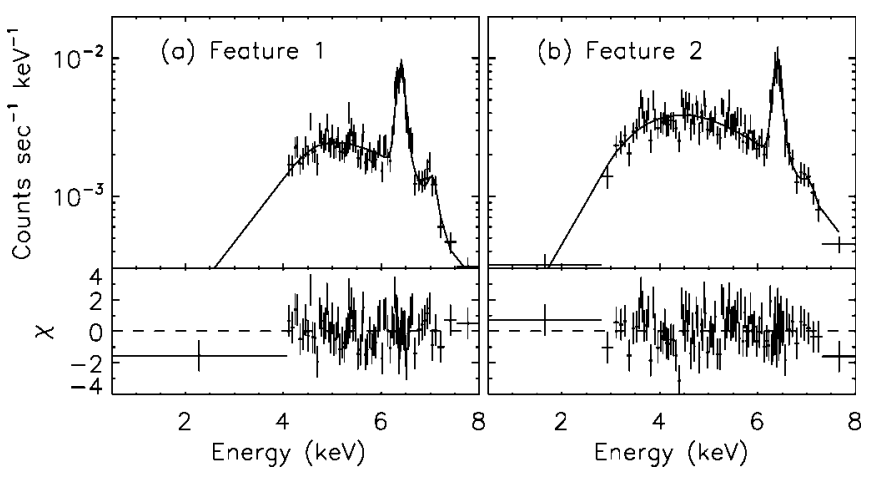

FIG. 3.-Spectra of fluorescent features 1 and 2. The top panels display the spectra in units of detector counts (crosses). The solid line is the best-fit model of an absorbed power law and two narrow lines at 6.4 and $7.0 \mathrm{keV}$. The bottom panels display the residuals, in units of the statistical uncertainty in the counts in each bin. The dominant feature in each spectrum is the iron line at $6.4 \mathrm{keV}$.

in the flux to the east of feature 2 and the south of feature 1. The pervasive nature of these variations is not surprising, because the entire field contains molecular gas (Tsuboi et al. 1999) and exhibits diffuse iron $\mathrm{K} \alpha$ emission (Muno et al. 2004).

Next, we extracted spectra of the diffuse features. The source spectra were extracted from the solid ellipses in Figure 1 (top) and the background from the dotted ellipses. We excluded events that fell within circles circumscribing the $92 \%$ encircled-energy contours of the PSFs for each point source. The backgroundsubtracted spectra were grouped so that each bin had a signal-tonoise ratio of $>5$. Spectra for all of the data are displayed in Figure 3.

We modeled the spectra from the 2002 and 2004-2005 epochs jointly using XSPEC, as a power-law continuum with photon index $\Gamma$ and normalization $N_{\Gamma}$, and two narrow iron lines at $6.40(\mathrm{Fe} \mathrm{K} \alpha)$ and $7.06 \mathrm{keV}(\mathrm{Fe} \mathrm{K} \beta)$. We assumed that this emission was absorbed by the interstellar medium $\left(N_{\mathrm{H}, \text { ISM }}\right)$, plus a component of the absorption intrinsic to the associated molecular clouds $\left(N_{\mathrm{H} \text {, cloud }}\right)$ that covered a variable fraction of the emitting region $\left(f_{\text {cloud }}\right)$. The use of such a "partial covering" factor $\left(f_{\text {cloud }}\right)$ was motivated by the assumption that the emission from the clouds would take place at a range of optical depths. We also assumed that $\Gamma$ and $N_{\mathrm{H} \text {, ISM }}$ were identical for both features during both epochs. In Table 1 we list the best-fit parameter values, iron equivalent widths, and fluxes.

Both features have extremely strong $\mathrm{Fe} \mathrm{K} \alpha$ emission, with equivalent widths of $\approx 1 \mathrm{keV}$ (Park et al. 2004). The Fe K $\beta$ emission is $\approx 10 \%-15 \%$ of the strength of the $\mathrm{K} \alpha$ line, as is expected for fluorescent emission (e.g., Murakami et al. 2000). Both features are heavily absorbed, so most of the observed flux is received between 4 and $8 \mathrm{keV}$. We find that for feature 1 , despite the noticeable changes in the morphology, the 4$8 \mathrm{keV}$ flux and the iron flux in the extraction region remained constant between 2002 and 2005. For feature 2, the inferred iron flux remained constant, but the observed $4-8 \mathrm{keV}$ flux declined by $26 \% \pm 7 \%$.

\section{DISCUSSION}

We have found that two fluorescent iron features located $6^{\prime}$ (14 pc in projection) from $\mathrm{Sgr} \mathrm{A}^{*}$ varied in morphology and intensity between 2002 and 2005 (Figs. 1 and 2 and Table 1). These changes require that the illuminating source varied on a timescale shorter than 3 years. Moreover, the features are $\approx 0.3^{\prime}$ wide and $\approx 1^{\prime}$ long, which corresponds to projected physical sizes of $\approx 0.7 \times 2$ pc. The smaller dimension constrains
TABLE 1

Spectra of the Iron Fluorescence Features

\begin{tabular}{|c|c|c|c|c|}
\hline \multirow[b]{2}{*}{ Parameter } & \multicolumn{2}{|c|}{ FEATURE I } & \multicolumn{2}{|c|}{ FEATURE 2} \\
\hline & 2002 & 2004-2005 & 2002 & 2004-2005 \\
\hline$N_{\mathrm{H}, \text { ISM }} \ldots \ldots \ldots$ & $11_{-1}^{+2}$ & $11^{\mathrm{a}}$ & $11^{\mathrm{a}}$ & $11^{\mathrm{a}}$ \\
\hline$N_{\mathrm{H}, \text { cloud }} \ldots \ldots$ & $25_{-2}^{+4}$ & $23_{-2}^{+4}$ & $29_{-6}^{+20}$ & $64_{-18}^{+26}$ \\
\hline$f_{\text {cloud }} \ldots \ldots \ldots$ & $1.0^{\mathrm{b}}$ & $1.0^{\mathrm{b}}$ & $0.61_{-0.05}^{+0.10}$ & $0.76_{-0.05}^{+0.08}$ \\
\hline$\Gamma \ldots \ldots \ldots$ & $1.84_{-0.13}^{+0.03}$ & $1.84^{\mathrm{a}}$ & $1.84^{\mathrm{a}}$ & $1.84^{\mathrm{a}}$ \\
\hline$N_{\Gamma} \ldots \ldots \ldots$ & $6_{-2}^{+2}$ & $5_{-2}^{+4}$ & $6_{-2}^{+1}$ & $8_{-2}^{+6}$ \\
\hline$F_{\mathrm{K} \alpha} \ldots \ldots \ldots$ & $18_{-1}^{+2}$ & $16_{-1}^{+1}$ & $19_{-2}^{+3}$ & $18_{-4}^{+8}$ \\
\hline $\begin{array}{lll}F_{\mathrm{K} \beta} \alpha & \ldots \ldots \ldots \ldots\end{array}$ & $2.1_{-0.6}^{+0.6}$ & $1.9_{-0.9}^{+0.9}$ & $1.5_{-0.8}^{+0.8}$ & $1.9_{-1.8}^{+1.7}$ \\
\hline $\mathrm{EW}_{\mathrm{K} \alpha} \ldots \ldots$ & $1000_{-90}^{+240}$ & $1010_{-90}^{+190}$ & $930_{-160}^{+160}$ & $690_{-220}^{+220}$ \\
\hline $\mathrm{EW}_{\mathrm{K} \beta} \ldots$ & $140_{-20}^{+70}$ & $150_{-70}^{+60}$ & $90_{-45}^{+40}$ & $90_{-80}^{+100}$ \\
\hline$F_{\mathrm{X}} \ldots \ldots \ldots \ldots$ & $4.0_{-0.2}^{+0.2}$ & $3.7_{-0.2}^{+0.2}$ & $5.7_{-0.3}^{+0.3}$ & $4.2_{-0.30}^{+0.3}$ \\
\hline$F_{\mathrm{X}}^{\prime} \ldots \ldots \ldots$ & $5.2_{-0.3}^{+0.2}$ & $4.8_{-0.3}^{+0.2}$ & $7.7_{-0.4}^{+0.5}$ & $5.5_{-0.5}^{+0.3}$ \\
\hline
\end{tabular}

Notes. - The reduced $\chi^{2}$ for the joint fit was $211 / 176$. The units are as follows: $N_{\mathrm{H}, \text { ISM }}$ and $N_{\mathrm{H} \text {, cloud }}$ are $10^{22} \mathrm{~cm}^{-2} ; N_{\Gamma}$ is $10^{-4}$ photons $\mathrm{cm}^{-2} \mathrm{~s}^{-1} \mathrm{keV}^{-1}$ at $1 \mathrm{keV} ; F_{\mathrm{K} \alpha}$ and $F_{\mathrm{K} \beta}$ are $10^{-6} \mathrm{ph} \mathrm{cm}^{-2} \mathrm{~s}^{-1}$; $\mathrm{EW}_{\mathrm{K} \alpha}$ and $\mathrm{EW}_{\mathrm{K} \beta}$ are eV; $F_{\mathrm{X}}$ is the observed 4-8 keV flux in units of $10^{-13} \mathrm{ergs} \mathrm{cm}^{-2} \mathrm{~s}^{-1}$; and $F_{\mathrm{X}}^{\prime}$ is the absorption-corrected $4-8 \mathrm{keV}$ flux in units of $10^{-13} \mathrm{ergs} \mathrm{cm}^{-2} \mathrm{~s}^{-1}$. Uncertainties are $1 \sigma$, for 1 degree of freedom for the parameters, and for 7 degrees of freedom for the fluxes and equivalent widths.

a The interstellar absorption and photon index of the continuum were assumed to be identical for each spectrum.

${ }^{\mathrm{b}}$ For feature $1, f_{\text {cloud }}$ was poorly constrained, so we fixed the parameter to its best-fit value of 1 .

the minimum duration of the outburst to have been 2 years. The fact that two features with a projected separation of $\approx 12$ 1t-yr varied over the same time interval implies that the illuminating source and the target clouds have separations with a component along our line of sight.

Such rapid changes over the course of a few years would not be produced by steady, slow-moving sources such as ejecta from supernovae (Bykov 2002). These shifting iron fluorescence features also would not be produced by $\approx 30 \mathrm{keV}$ electrons, which would have velocities of only $0.06 c$ (and slower diffusion speeds). The most reasonable explanation for these variable fluorescent features is that they are scattered emission from the outburst of a transient hard X-ray source, such as an X-ray binary or the supermassive black hole Sgr A*.

The luminosity of the source illuminating these reflection nebulae can be estimated from the optical depths of the scattering media coupled with the fluxes in the iron lines. The luminosity in the fluorescent $\mathrm{Fe} \mathrm{K} \alpha$ line is about $30 \%$ of the flux absorbed by the photoelectric edge, and so the observed flux can be computed from

$$
F_{\mathrm{K} \alpha}=\frac{0.3}{4 \pi D^{2}} \int_{E_{0}}^{\infty} \frac{\mathcal{L}_{\mathrm{X}}(E)}{4 \pi d^{2}} A\left(1-e^{-\tau_{\mathrm{Fe}}\left(E / E_{0}\right)^{-3}}\right) d E,
$$

where $D$ is the distance from the observer to the fluorescing cloud, $\mathcal{L}_{\mathrm{X}}(E)$ is the specific luminosity of the illuminating source as a function of energy (units of photons $\mathrm{s}^{-1} \mathrm{keV}^{-1}$ ), $d$ is the distance between the source and the cloud, $A$ is the area of the cloud projected toward the source, and $\tau_{\mathrm{Fe}}$ is the optical depth of the iron photoelectric edge at an energy $E_{0}=7.1 \mathrm{keV}$.

This equation can be simplified given the parameters of our nebulae. The optical depth is given by $\tau_{\mathrm{Fe}}=N_{\mathrm{H}} \delta_{\mathrm{Fe}} \sigma_{\mathrm{Fe}}$, where $\sigma_{\mathrm{Fe}}=3.5 \times 10^{-20} \mathrm{~cm}^{2}$ is the cross section for photoelectric absorption at $7.1 \mathrm{keV}$, and $\delta_{\mathrm{Fe}}=3 \times 10^{-5}$ is the fractional abundance of iron for solar abundances. If we take $N_{\mathrm{H}} \lesssim 6 \times 10^{23} \mathrm{~cm}^{-2}$ (Table 1), the optical depth to iron absorption is $\tau_{\mathrm{Fe}} \lesssim 0.8$, and $1-e^{-\tau} \approx \tau$ with an error of $<30 \%$. Next, we take $D=8 \mathrm{kpc}$, define $d_{10}$ to be normalized to $10 \mathrm{pc}$, 
rewrite $A$ as a solid angle $\Omega_{1}$ normalized to $1 \operatorname{arcmin}^{2}$ at $8 \mathrm{kpc}$, and write $\tau_{\mathrm{Fe}}$ in terms of the column depth $N_{\mathrm{H}, 23}$ in units of $10^{23} \mathrm{~cm}^{-2}$ and the abundance of iron relative to the solar value $\alpha_{\mathrm{Fe}}$. Finally, we assume that the spectrum of the illuminating source is a $\Gamma=1.8$ power law, $\mathcal{L}_{\mathrm{X}}(E) \propto E^{-1.8}$. We then can integrate equation (1) over energy and write the result in terms of the 2-8 $\mathrm{keV}$ luminosity, normalized to $10^{38} \mathrm{ergs} \mathrm{s}^{-1}, L_{\mathrm{X}, 38}$. With these substitutions, we find

$$
F_{\mathrm{K} \alpha}=5 \times 10^{-5} L_{\mathrm{X}, 38} d_{10}^{-2} N_{\mathrm{H}, 23} \alpha_{\mathrm{Fe}} \Omega_{1} \text { photons } \mathrm{cm}^{-2} \mathrm{~s}^{-1} .
$$

If $\mathrm{Sgr} \mathrm{A}^{*}$ is the source of the photons that produced the reflection features, and the line-of-sight separation between it and the cloud is equal to the projected separation (14 pc), $d \approx 20 \mathrm{pc}$ and an outburst with $L_{\mathrm{X}} \sim 10^{38} \mathrm{ergs} \mathrm{s}^{-1}$ would have been required. ${ }^{6}$ Such an outburst would have occurred 60 years ago, before the advent of X-ray astronomy, and therefore would not have been observed. The lack of similar reflection nebula at negative Galactic longitudes (Park et al. 2004) implies that those clouds do not lie within the light front of the outburst.

Alternatively, if the bright transient that was in outburst in 2004 produced the fluorescent features, $d \approx 7 \mathrm{pc}$ and $L_{\mathrm{X}} \sim$ $10^{37} \mathrm{ergs} \mathrm{s}^{-1}$. Although this is 100 times brighter than the outbursts in 2002 and 2004 (Porquet et al. 2005; Muno et al. 2005), this outburst would have occurred 20 years ago, before the advent of both Chandra and the wide-field X-ray monitors on BeppoSAX and the Rossi X-Ray Timing Explorer, so it too could have been missed. However, it is uncommon for the outburst of an X-ray binary to persist at this luminosity for 23 years (Chen et al. 1997), ${ }^{7}$ so we prefer the hypothesis that the source of the outburst was Sgr A*.

The time evolution of a reflection nebula has been explored in detail by Sunyaev \& Churazov (1998) assuming target material with a spherical geometry. However, the observed changes in the morphology in Figure 1 are significantly more complex than they considered. For instance, in feature 1 the

\footnotetext{
${ }^{6}$ This luminosity is larger than that reported in Park et al. (2004) because of an error in the earlier paper.

${ }^{7}$ See also http://xte.mit.edu/ASM_lc.html.
}

vertical filament $\approx 2 \mathrm{pc}$ long fades over the course of 3 years. The fact that the light-travel time along the feature is longer than the interval over which it fades implies that the light front propagated perpendicular to the filament and had width that is similar to that of the filament $(0.3 \mathrm{pc})$. The fact that the whole filament dimmed, with very little brightening adjacent to most of its length (aside from knots in the north on either side of the filament), implies that the X-rays were no longer propagating through dense material in 2004 and 2005. Therefore, the clouds must contain filamentary features a few tenths of a parsec wide and a couple parsecs long.

\section{CONCLUSIONS}

The changes in the intensity and morphology of fluorescent iron nebulae near the Galactic center demonstrates that they are reflection nebulae. The most likely source of the reflected $\mathrm{X}$-rays is an outburst of $\mathrm{Sgr} \mathrm{A}^{*}$ with a luminosity of at least $10^{38} \mathrm{ergs} \mathrm{s}^{-1}$, which would have occurred about 60 years ago and lasted 2-3 years. If a similar outburst had occurred since then, it would have had a good chance of being detected by an X-ray instrument or would have produced similar fluorescent features in other, nearby clouds. Since no other long outburst of $\mathrm{Sgr} \mathrm{A}^{*}$ has been reported, and no fluorescent features have been identified closer to Sgr A* (Park et al. 2004), the duty cycle of bright, year-long outbursts from $\operatorname{Sgr~}^{*}$ must be $\$ 5 \%$.

These fluorescent features also reveal filamentary structures within the dense regions of the molecular clouds. Interferometric millimeter observations would resolve clouds on a similar spatial scale and also provide velocity information. Combining the cloud velocities and the three-dimensional spatial information afforded by the evolution of the reflection nebulae would allow one to construct a physical model of these turbulent molecular clouds. This is a necessary step toward modeling star formation in the hot, turbulent environment of the Galactic center.

This work was funded by NASA through the Chandra $X$ Ray Observatory.

\section{REFERENCES}

Bykov, A. M. 2002, A\&A, 390, 327

Chen, W., Shrader, C. R., \& Livio, M. 1997, ApJ, 491, 312

Gehrels, N. 1986, ApJ, 303, 336

Koyama, K., Maeda, Y., Sonobe, T., Takeshima, T., Tanaka, Y., \& Yamauchi, S. 1996, PASJ, 48, 249

McNamara, D. H., Madsen, J. B., Barnes, J., \& Ericksen, B. F. 2000, PASP, 112,202

Muno, M. P., Pfahl, E., Baganoff, F. K., Brandt, W. N., Ghez, A., Lu, J., \& Morris, M. R. 2005, ApJ, 622, L113

Muno, M. P., et al. 2004, ApJ, 613, 326

Murakami, H., Koyama, K., \& Maeda, Y. 2001b, ApJ, 558, 687

Murakami, H., Koyama, K., Sakano, M., Tsujimoto, M., \& Maeda, Y. 2000, ApJ, 534, 283

Murakami, H., Koyama, K., Tsujimoto, M., Maeda, Y., \& Sakano, M. 2001a, ApJ, 550, 297
Park, S., Muno, M. P., Baganoff, F. K., Maeda, Y., Morris, M., Howard, C., Bautz, M. W., \& Garmire, G. P. 2004, ApJ, 603, 548

Porquet, D., Grosso, N., Burwitz, V., Andronov, I. L., Aschenbach, B., Predehl, P., \& Warwick, R. S. 2005, A\&A, 430, L9

Revnivtsev, M. G., et al. 2004a, Astron. Lett., 30, 382

- 2004b, A\&A, 425, L49

Skrutskie, M. F., et al. 2006, AJ, 131, 1163

Sunyaev, R., \& Churazov, E. 1998, MNRAS, 297, 1279

Sunyaev, R. A., Markevitch, M., \& Pavlinsky, M. 1993, ApJ, 407, 606

Tsuboi, M., Handa, T., \& Ukita, N. 1999, ApJS, 120, 1

Valinia, A., Tatischeff, V., Arnaud, K., Ebisawa, K., \& Ramaty, R. 2000, ApJ, 543,733

Yusef-Zadeh, F., Law, C., \& Wardle, M. 2002, ApJ, 568, L121 